\title{
Article \\ Comprehensive Genomic Characterization of Fifteen Early-Onset Lynch-Like Syndrome Colorectal Cancers
}

\author{
Mariano Golubicki ${ }^{1,2} \mathbb{D}^{\mathbb{D}}$, Marcos Díaz-Gay $^{3,4}{ }^{\mathbb{D}}$, Laia Bonjoch ${ }^{3} \mathbb{D}$, Sebastià Franch-Expósito ${ }^{3}$, Jenifer Muñoz ${ }^{3}$, \\ Miriam Cuatrecasas ${ }^{5}$ D, Teresa Ocaña ${ }^{3}$, Soledad Iseas ${ }^{1}$, Guillermo Mendez ${ }^{1}$, Marcela Carballido ${ }^{1}$, \\ Juan Robbio ${ }^{1,2} \mathbb{D}$, Daniel Cisterna ${ }^{2} \mathbb{D}$, Enrique Roca ${ }^{1}$, Antoni Castells ${ }^{3} \mathbb{D}$, Francesc Balaguer ${ }^{3}$ (D), \\ Sergi Castellví-Bel ${ }^{3, *(\mathbb{D})}$ and Marina Antelo ${ }^{1, *}$
}

check for updates

Citation: Golubicki, M.; Díaz-Gay, M.; Bonjoch, L.; Franch-Expósito, S.; Muñoz, J.; Cuatrecasas, M.; Ocaña, T.; Iseas, S.; Mendez, G.; Carballido, M.; et al. Comprehensive Genomic Characterization of Fifteen Early-Onset Lynch-Like Syndrome Colorectal Cancers. Cancers 2021, 13, 1259. https://doi.org/10.3390/ cancers13061259

Academic Editor: Frank A. Simmen

Received: 28 January 2021

Accepted: 9 March 2021

Published: 12 March 2021

Publisher's Note: MDPI stays neutral with regard to jurisdictional claims in published maps and institutional affiliations.

Copyright: (c) 2021 by the authors. Licensee MDPI, Basel, Switzerland. This article is an open access article distributed under the terms and conditions of the Creative Commons Attribution (CC BY) license (https:/ / creativecommons.org/licenses/by/ $4.0 /)$.
1 Oncology Section, Hospital of Gastroenterology “Dr. C. B. Udaondo”, Buenos Aires C1264, Argentina; mariano.golubicki@gmail.com (M.G.); soledad.iseas@gmail.com (S.I.); mendezdoc@hotmail.com (G.M.); mcarballido@gmail.com (M.C.); robbiojuan@gmail.com (J.R.); enlroca@yahoo.com.ar (E.R.)

2 Molecular Biology Lab, Hospital of Gastroenterology “Dr. C. B. Udaondo”, Buenos Aires C1264, Argentina; dancis99@yahoo.com

3 Centro de Investigación Biomédica en Red de Enfermedades Hepáticas y Digestivas (CIBERehd), Gastroenterology Department, Institut d' Investigacions BiomèdiquesAugust Pi i Sunyer (IDIBAPS), Hospital Clínic, 08036 Barcelona, Spain; mdiazgay@health.ucsd.edu (M.D.-G.); bonjoch@clinic.cat (L.B.); franches@mskcc.org (S.F.-E.); jenifer.munoz@ciberehd.org (J.M.); mocana@clinic.cat (T.O.); castells@clinic.cat (A.C.); fprunes@clinic.cat (F.B.)

4 Department of Cellular and Molecular Medicine, University of California, San Diego, La Jolla, CA 92093, USA

5 Pathology Department, Hospital Clínic, 08036 Barcelona, Spain; mcuatrec@clinic.cat

* Correspondence: sbel@clinic.cat (S.C.-B.); machuantelo@hotmail.com (M.A.)

Simple Summary: The most prevalent type of hereditary colorectal cancer is called Lynch syndrome and it is characterized by a tumor phenotype called microsatellite instability (MSI). This disease is a consequence of germline (inheritable) variants in any of the four mismatch repair (MMR) DNA genes, being their identification essential to ensure their appropriate diagnosis and implementation of preventive measurements. Nevertheless, only 50\% of patients with MSI and suspected Lynch syndrome actually carry a germline pathogenic variant in an MMR gene that explains the clinical entity. The remaining 50\% are termed Lynch-like syndrome, and their causes remain unknown. In this work, we tried to elucidate the molecular mechanisms that underlie this rare entity in a group of early-onset Lynch-like syndrome colorectal cancer, through whole-exome sequencing of germline and tumor samples. We observed that one-third of these patients have somatic alterations in genes associated with the MMR system and that these could be the mechanism causing their unexplained MSI. Furthermore, we found that patients who showed biallelic somatic alterations also carried germline variants in new candidate genes associated with DNA repair functions and that this could be, partly, the cause of the early onset in this cohort.

Abstract: Lynch-like syndrome (LLS) is an increasingly common clinical challenge with an underlying molecular basis mostly unknown. To shed light onto it, we focused on a very young LLS early-onset colorectal cancer (CRC) cohort (diagnosis $\leq 40$ y.o.), performing germline and tumor whole-exome sequencing (WES) of 15 patients, and additionally analyzing their corresponding tumor mutational burden (TMB) and mutational signatures. We identified four cases (27\%) with double somatic putative variants in mismatch repair (MMR) core genes, as well as three additional cases (20\%) with double $\mathrm{MSH} 3$ somatic alterations in tumors with unexplained MSH2/MSH6 loss of expression, and two cases $(13 \%)$ with POLD1 potential biallelic alterations. Average TMB was significantly higher for LLS cases with double somatic alterations. Lastly, nine predicted deleterious variants in genes involved in the DNA repair functions and/or previously associated with CRC were found in nine probands, four of which also showed MMR biallelic somatic inactivation. In conclusion, we contribute new insights into LLS CRC, postulating MSH3 and POLD1 double somatic alterations as an underlying cause of a microsatellite instability (MSI) phenotype, proposing intrinsic biological differences between LLS with and without somatic alterations, and suggesting new predisposing candidate genes in this scenario. 
Keywords: colorectal cancer; biallelic somatic alteration; early-onset cancer; Lynch-like syndrome; mismatch repair; whole-exome sequencing

\section{Introduction}

Colorectal cancer (CRC) is the second most commonly diagnosed cancer and the third leading cause of cancer deaths in most developed countries, with a mean age of 70 years at diagnosis [1]. However, up to $15 \%$ of all cases occur before the age of 50 years [2], and recent epidemiological studies suggest that incidence and mortality of these early-onset CRCs are increasing [3,4]. Several hereditary (Lynch syndrome, familial adenomatous polyposis, MUTYH-associated polyposis) and non-hereditary diseases (ulcerative colitis, Crohn's disease) are associated with early-onset CRC, and their early detection is essential because of the increased lifetime CRC risk and the potential positive impact of preventive measures on survival [2]. Inflammatory bowel disease and colonic polyposis syndromes are easily identified from their phenotypic features, but Lynch syndrome patients do not have a characteristic clinical phenotype and are often missed, especially in the absence of a family history of cancer.

Lynch syndrome (LS) is the most common hereditary cancer syndrome, affecting an estimated 1 in 300 individuals and have reported to have increased incidences of cancers in the colon, rectum, endometrium, ovaries, stomach, small bowel, bile duct, pancreas, and upper urinary tract. LS is also the most common hereditary cause of CRC, accounting for approximately $1-4 \%$ of all cases [5]. It is an autosomal dominant condition caused by germline pathogenic or likely pathogenic variants in a DNA mismatch repair (MMR) gene. MSH2 and MLH1 account for most Lynch syndrome-associated CRCs. This syndrome has a marked genetic-dependent variable penetrance for CRC and endometrial carcinoma (30-80\%), and patients are at increased risk for other extra-colonic tumors. Annual surveillance colonoscopies and total hysterectomy reduce cancer mortality [6]. Additionally, the identification of a causal deleterious variant in one of the MMR genes leads to genetic pre-symptomatic diagnosis in relatives, focusing screening measures on variant carriers.

While the hallmark of this disease is tumor MMR deficiency (MMRd), defined by the presence of microsatellite instability (MSI) and/or absence of MMR protein expression by immunohistochemistry (IHC), BRAF V600E wild type, and no MLH1 promoter hypermethylation, a diagnosis of Lynch syndrome requires the presence of a deleterious germline variant in a DNA MMR gene [7]. MMRd tumors without a germline variant in any of the four MMR genes may account for as many as $70 \%$ of cases [8]. These cases are termed "Lynch-like syndrome" (LLS), and management decisions in these patients and their families are complicated because of unconfirmed suspicions of hereditary cancer [9]. In addition to the hypothesis regarding possible "cryptic" deleterious germline variants in MMR genes, there are two other possible explanations proposed: unknown germline pathogenic or likely pathogenic variants affecting other than MMR genes may drive the tumor towards MSI (as it has been recently described for MUTYH and POLE genes in isolated LLS cases [10-12]), and biallelic somatic inactivation of an MMR gene in LLS tumors may cause MMRd (as it has been suggested in several reports [13-16]).

Besides the MMR genes, the wider LLS tumors' somatic alteration profiles are unfamiliar, and there is actually scant information about underlying somatic characteristics and the complex processes behind the MMR protein inactivation. To shed light onto it, we thus focused on a very young LLS early-onset CRC cohort to elucidate the underlying molecular basis of this increasingly common clinical challenge, by performing germline and tumor whole-exome sequencing (WES) of 15 patients with early-onset LLS CRC. 


\section{Results}

\subsection{Patients}

Fifteen patients with CRC LLS diagnosed $\leq 40$ y.o. were included in this study. Demographic, clinic-histopathological, and MMR characterization of this cohort is presented in Table 1. Mean age at diagnosis was 30.2 years (SD 7.2); 9 (60\%) were women, and 11 $(73 \%)$ patients had tumors located distal to the splenic flexure. The majority of cases (12; $80 \%$ ) were diagnosed at advanced stages (III-IV). Lack of MLH1/PMS2 expression was as frequent as MSH2/MSH6 (both 7/15; 47\% each). Isolated loss of MLH1 and MSH6 (2/15; $0.7 \%$ each) were less frequent. All patients fulfilled Bethesda 1 criteria, and half of them also presented MSI-suggestive histology (Bethesda 3 criteria).

\subsection{Somatic Biallelic Alterations in MMR Core and MMR-Associated Genes}

When exploring somatic alterations in the MMR core genes (MLH1, MSH2, MSH6, PMS2), four of 15 patients (27\%) had two somatic heterozygous variants explaining their IHC findings, one in MSH2 (LLS18) and three in MLH1 (LLS17, LLS21, LLS9045). Seven of these variants were truncating and potentially pathogenic; although the remaining one was a missense variant (p.Gly67Arg), Clinvar and three in silico prediction tools classified it as pathogenic.

On the other hand, when MMR-associated genes (MSH3, MLH3, PMS1, MUTYH, POLE, POLD1) were also considered, we observed five additional cases (33\%) with putative biallelic alterations. Three patients with IHC MSH2/MSH6 loss of expression (LLS02, LLS09, LLS10) showed one somatic MSH3 truncating variant and associated LOH (considered as a second hit). Two additional patients were found to carry biallelic POLD1 potentially pathogenic variants with MSH2/MSH6 and MLH1/PMS2 loss of expression, respectively (LLS9049, LLS5159). Of note, LLS10 showed concomitant MSH3 and POLD1 biallelic alterations.

Lastly, LLS9045 (which showed MLH1 biallelic somatic variants), also presented biallelic alterations in $M L H 3$ (a missense variant with associated LOH).

In the remaining six patients $(40 \%)$, no biallelic somatic alterations were identified in the analyzed genes. Of these, 3 showed MLH1/PMS2 loss of expression (LLS15, LLS5604), one isolated MSH6 deficiency (LLS06), and two MSH2/MSH6 (LLS15, LLS20).

All results are presented in Figure 1 and all somatic findings are summarized in Table 2. The InSiGHT database classifications (http: / / www.insight-database.org/classifications / access date: 16 January 2021) for all MMR variants are shown in Table S4. Additionally, all somatic variants classified according to the ACMG criteria as pathogenic, likely pathogenic, uncertain significance, likely benign and benign, are shown in Table S5. 
Table 1. Clinical and-pathological features of the colorectal cancer (CRC) Lynch-like syndrome cohort.

\begin{tabular}{|c|c|c|c|c|c|c|c|c|c|c|c|c|c|}
\hline Patient ID & Cohort & $\begin{array}{c}\text { Age of Onset } \\
\text { (Years) }\end{array}$ & Sex & Tumor Location & $\begin{array}{l}\text { CRC Relation to } \\
\text { Splenic Flexure }\end{array}$ & Stage & $\begin{array}{c}\text { Histological } \\
\text { Differentiation }\end{array}$ & $\begin{array}{l}\text { Bethesda } \\
\text { Clinical } \\
\text { Criteria }\end{array}$ & MSI Status & $\begin{array}{c}\text { MMR } \\
\text { Protein Loss }\end{array}$ & $\begin{array}{c}\text { Somatic } \\
\text { Braf v600e }\end{array}$ & $\begin{array}{l}\text { Biallelic Somatic } \\
\text { Alteration Gene }\end{array}$ & $\begin{array}{l}\text { TMB (Muta- } \\
\text { tions/mb) }\end{array}$ \\
\hline LLS02 & Udaondo & 12 & male & rectum & Left & IV & well & 1 & MSI & MSH2/MSH6 & WT & MSH3 & 26.7 \\
\hline LLS06 & Udaondo & 30 & female & ascending colon & Right & IIIA & moderately & $1 ; 5$ & MSS & MSH6 & WT & no alteration & 9.4 \\
\hline LLS09 & Udaondo & 30 & female & rectum & Left & IIIA & moderately & $1 ; 5$ & MSI & MSH2/MSH6 & WT & MSH3 & 81.2 \\
\hline LLS10 & Udaondo & 25 & female & descending colon & Left & IIB & moderately & $1 ; 3$ & MSI & MSH2/MSH6 & WT & MSH3; POLD1 & 118 \\
\hline LLS15 & Udaondo & 19 & male & descending colon & Left & IIA & no data & $1 ; 3$ & MSI & MLH1/PMS2 & WT & no alteration & 44.7 \\
\hline LLS17 & Udaondo & 40 & male & descending colon & Left & IIIB & poorly & $1 ; 3$ & MSI & MLH1/PMS2 & WT & MLH1 & 77 \\
\hline LLS18 & Udaondo & 30 & female & descending colon & Left & IIA & moderately & $1 ; 3$ & MSI & MSH2/MSH6 & WT & MSH2 & 57.8 \\
\hline LLS19 & Udaondo & 25 & female & caecum & Right & IIIA & well & $1 ; 2 ; 3$ & MSI & MSH2/MSH6 & WT & no alteration & 163.3 \\
\hline LLS20 & Udaondo & 33 & male & ascending colon & Right & IIIA & moderately & 1 & MSI & MSH2/MSH6 & WT & no alteration & 7.6 \\
\hline LLS9049 & Clinic & 31 & female & descending colon & Left & $\mathrm{IIIB}$ & moderately & 1 & MSI & MSH2/MSH6 & WT & POLD1 & 164.6 \\
\hline LLS9045 & Clinic & 35 & male & rectum & Left & IIIB & moderately & 1 & MSI & MLH1/PMS2 & WT & MLH1 & 142.4 \\
\hline LLS5159 & Clinic & 37 & female & sigma & Left & IV & poorly & $1 ; 3$ & MSI & MLH1/PMS2 & WT & POLD1 & 81.4 \\
\hline LLS5604 & Clinic & 33 & male & caecum & Right & IIIB & poorly & $1 ; 3$ & MSI & MLH1/PMS2 & WT & no alteration & 16.3 \\
\hline LLS9048 & Clinic & 30 & male & ascending colon & Right & IIA & poorly & $1 ; 3$ & MSI & MLH1 & WT & no alteration & 6.8 \\
\hline
\end{tabular}

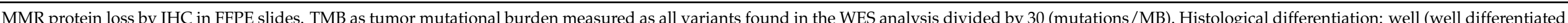

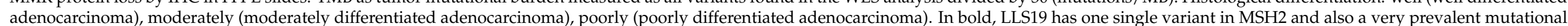
signature related to a previous alkylating agent treatment. 

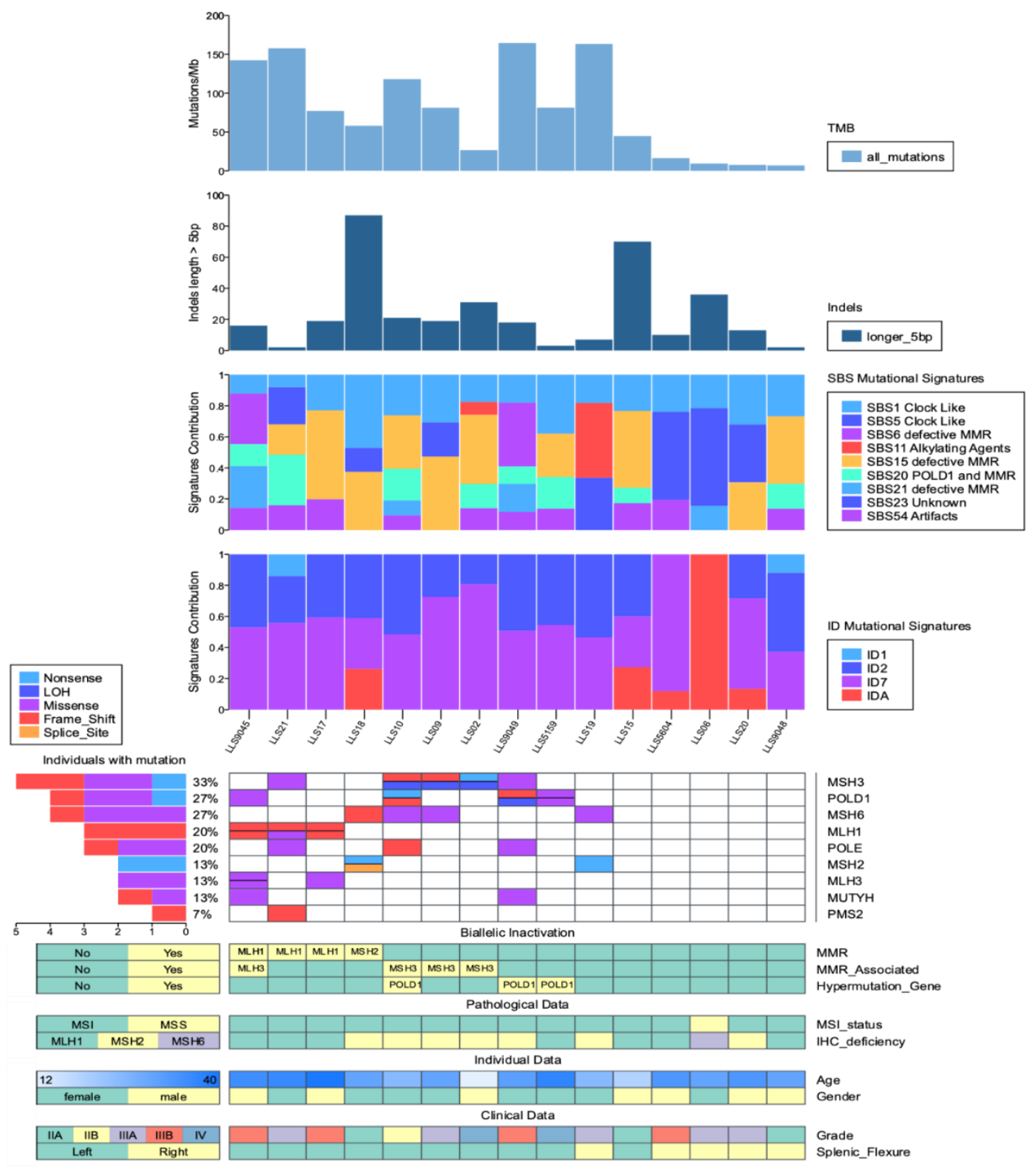

Figure 1. Genomic landscape of 15 early-onset colorectal cancer LLS patients. Samples are organized from left to right as MMR core genes biallelic inactivation, MMR-associated inactivation and hypermutated genes inactivation. Abbreviations $\mathrm{TMB}=$ tumor mutational burden (mutations / megabase); $\mathrm{SBS}=$ single base substitution; ID/Indels = short insertions and deletions; $\mathrm{LOH}=$ loss of heterozygosity. 
Table 2. Somatic bi- and monoallelic variants in core and associated MMR genes.

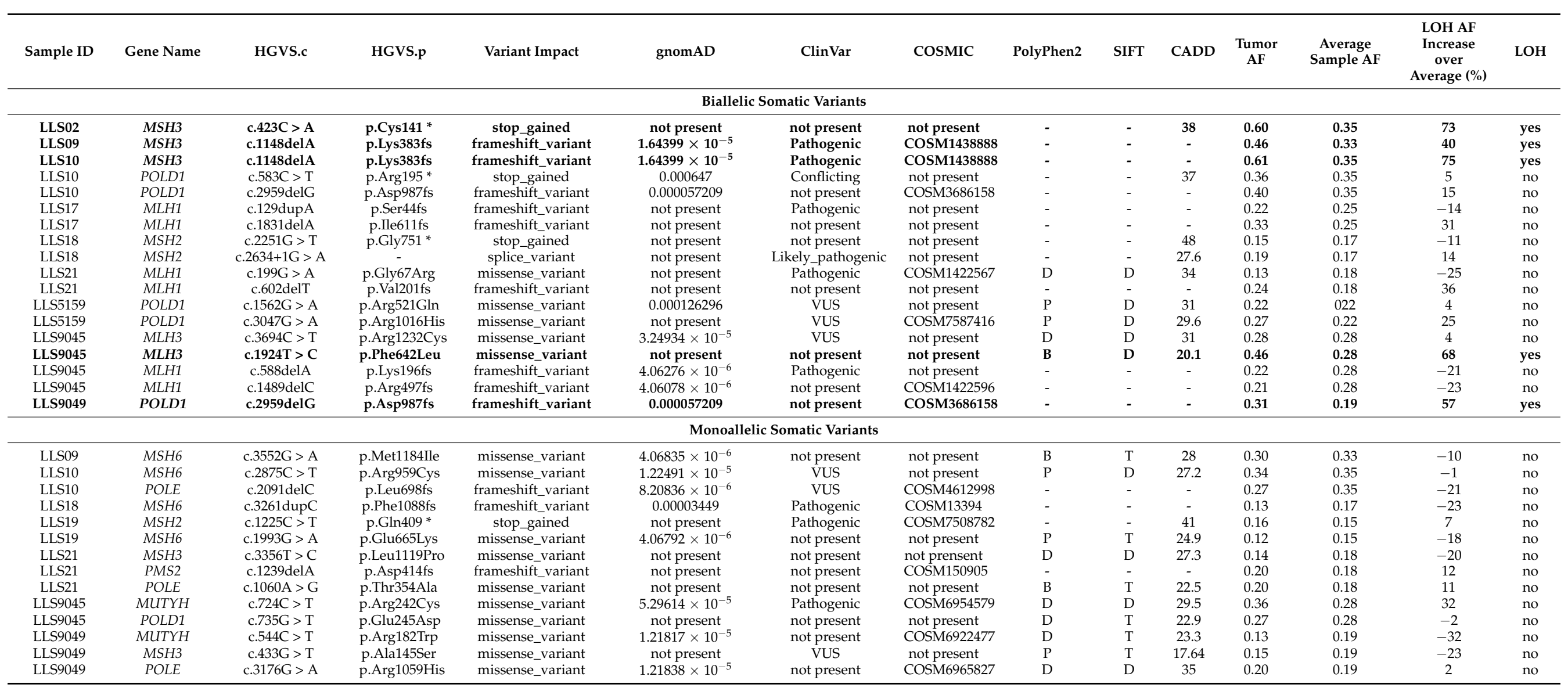

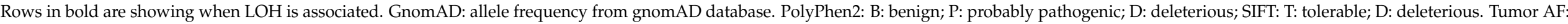

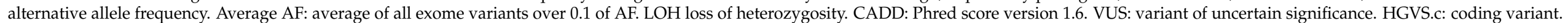
HGVS.p: protein level variant. * stop gained codon. 


\subsection{Somatic Monoallelic Alterations in MMR Core and MMR-Associated Genes}

We then evaluated monoallelic variants. In patients with no potential biallelic inactivation of MMR core and MMR associated gen, only LLS19, with MSH2 loss of IHC expression, showed single MSH2 and MSH6 variants, with no associated LOH.

On the other hand, additional monoallelic variants were identified in patients with biallelic inactivation, and are summarized in Figure 1 and Table 2.

\subsection{Somatic Alterations in Additional Cancer Genes}

Additional data on the most common CRC mutated genes are reported in Figure S1. $A P C$ and FBXW7 were mutated in $7 / 15$ cases (47\%), ATM and KRAS in 6/15 (40\%), and TP53 and PTEN showed variants in 2/15 (13,3\%). One single patient presented one variant in $S M A D 4$, and no variants were found in NRAS.

\subsection{MSH3 and MSI Association}

According to our results, we hypothesized that $\mathrm{MSH} 3$ biallelic somatic inactivation could be involved in LLS syndrome associated CRC. We therefore investigated if MSH3 variants could be associated with MSI in 3806 CRC cases with somatic profiling data from cBioPortal (http:/ / www.cbioportal.org access date: 16 January 2021). Although we observed 67 CRCs with MSH3 variants, only 13 (13/3806, 0.34\%) had at least one truncating MSH3 somatic pathogenic variant with no associated variants in $M L H 1, M S H 2, M S H 6$, or PMS2, nor MLH1 silencing by hypermethylation. Among these 13 cases, $85 \%(11 / 13)$ were MSI, and although one case had no MSI data, the number of variants was similar to those displaying MSI (sample ID: coadread_dfci_2016_60 and 1,937 variants). The remaining case $(1 / 13,8 \%)$ was reported as microsatellite stable (MSS) (Table S1).

\subsection{Tumor Mutational Burden (TMB)}

Five out of 15 patients (33.3\%) showed mutational burdens compatible with ultrahypermutation phenotype, accounting for over 100 mutations per megabase, and four of them $(4 / 5,80 \%)$ were patients with biallelic inactivation. Seven additional patients displayed a hypermutated phenotype with 10-100 mutations per megabase, and the remaining three were non-hypermutated, with less than 10 mutations per megabase (Table 1 and Figure 1).

Searching for molecular differences between patients with double somatic alterations in MMR genes, MSH3 or POLD1, and those with no somatic inactivation, we measured TMB and compared it according to these two groups. Interestingly, mean TMB of the first group was significantly higher (100.8 versus 17.0 mutations/Mb; Wilcoxon rank-sum test; $p$-value $=0.002)$. For this analysis, we excluded the second group LLS19 sample, which presented a particular mutational signature related to the patient's history of previous temozolomide treatment (see Section 2.7).

We then compared TMB according to tumor location, observing that patients with tumors distal to splenic flexure presented higher mutations per megabase than those proximal to splenic flexure (88.3 versus 8.5 mutations $/ \mathrm{Mb}$; Wilcoxon rank-sum test; $p$-value $=0.0019$ ) (Figure S2).

\subsection{Mutational Signatures}

Further somatic characterization was performed by analyzing mutational signatures, considering single base substitutions (SBS) and small insertion and deletions (ID), in order to have a closer look at the different mutational mechanisms implicated in our cohort. De novo extraction considering SNVs revealed six active mutational signatures, subsequently decomposed into nine previously indexed in version 3.1 of COSMIC database reference set (Jun 2020) (Figure S3) [17,18].

One of the previously known mutational signatures found in our cohort was SBS11, associated with temozolomide (TMZ) chemotherapy treatment. This signature was found as the major contributor in the LLS19 patient's CRC, who reported a previous TMZ 
treatment for a non-Hodgkin lymphoma diagnosed at the age of 21 years, four years prior to the MSI CRC diagnosis.

Besides this particularity, and as expected, the rest of the mutational signature contributions in the cohort were dominated by those related to MMR deficiency (45.1\% on average), with up to four different signatures present (SBS6, SBS15, SBS20, and SBS21). Interestingly, all nine tumors harboring somatic biallelic inactivation in MMR, MMR associated, or hypermutation genes showed a significantly higher number of mutations linked to MMR deficiency signatures versus those with monoallelic or no somatic mutations (1795.9 vs. 206.8 mean mutations; Wilcoxon sum-rank test; $p$-value $=0.004$; excluding LLS19 case).

In the case of small insertions and deletions (indels), four different mutational signatures were extracted and decomposed, corresponding to three known COSMIC v3.1 signatures related to MMR deficiency (ID1, ID2, and ID7), and one new signature that had not been previously reported (referred to as IDA) (Figure S3). This new signature is characterized by de novo insertions of 5 or more nucleotides. Interestingly, signature IDA was found active in four out of the 5 tumors without biallelic inactivation, whereas only in one of the inactivated samples (11\%). Indeed, the indel spectrum of the only case presenting a MSS tumor (and isolated loss of MSH6 by IHC), LLS06, is exclusively composed by IDA-linked alterations.

\subsection{Germline Candidate Genes}

In order to explore the hypothesis that somatic inactivation of MMR system could be a random consequence of deleterious germline variants in unknown genes, we analyzed dominant candidates and chose those with specific DNA repair functions from our WES data. A recessive candidate from this cohort was recently published [19].

All germline DNA samples were sequenced with good quality, with a mean coverage higher than $60 \times$. This prioritization strategy yielded 10 very rare predicted deleterious variants in 9 DNA repair associated genes (gnomAD $<0.1 \%$ or not present), and all of them scored over 20 in CADD Phred score. Results are summarized in Table 3.

Ten patients $(66.7 \%)$ carried predicted deleterious variants in 9 genes with functions involved in DNA repair mechanism; five patients without somatic biallelic variants carried five of these variants: four missense variants in ERCC6, POLE, EXO5, and RAD52, and one in a donor splice site in RECQL4. The remaining five variants were identified in patients with biallelic somatic inactivation: four missense in PALB2, UVRAG, RAD54L, and MCM2, and one in the acceptor splice site in RAD54L. Nine variants were cataloged as a VUS (variant of uncertain significance) by ACMG/AMP criteria, and one in RECQL4(c.1878+1G $>C$ ) was scored as pathogenic, disrupting the donor splice site of the exon 11. Results are summarized in Table 3.

The most promising candidate genes were POLE, ERCC6, RAD54L, and PALB2. The POLE variant (c.1847G > A; p.Arg616His) identified in LLS20 was outside the exonuclease domain, and its respective tumor was not hypermutated and did not present any mutational signatures previously associated with POLE variants. The ERCC6 variant (c.1670G > A, p.Arg557His) affecting the helicase ATP-binding domain was present in LLS06, whose tumor was MSS, showed low TMB, and no contribution of MMR deficiency-associated ID mutational signatures (Figure 1). 
Table 3. Germline candidate variants. Results of the whole exome sequencing analysis with candidate variants with DNA repair functions.

\begin{tabular}{|c|c|c|c|c|c|c|c|c|}
\hline ID & $\begin{array}{c}\text { Germline } \\
\text { Candidate } \\
\text { Gene }\end{array}$ & HGVS.c & HGVS.p & Coding Impact & gnomAD & CADD & ClinVar & ACMG Classification \\
\hline \multicolumn{9}{|c|}{ Patients without biallelic inactivation in MMR core or their associated genes } \\
\hline LLS06 & ERCC6 & c. $1670 \mathrm{G}>\mathrm{A}$ & R557H (p.Arg557His) & missense & 0.00018 & 27.6 & Not present & VUS(PM1;PM2;PP3;BP1) \\
\hline LLS20 & POLE & c. $1847 \mathrm{G}>\mathrm{A}$ & R616H (p.Arg616His) & missense & 0.0000358 & 26.4 & Not present & VUS (PM2;PP3;BP1) \\
\hline LLS5604 & EXO5 & c. $23 \mathrm{~A}>\mathrm{G}$ & E8G (p.Glu8Gly) & missense & Not present & 25.3 & Not present & VUS (PM2;BP4) \\
\hline LLS9048 & RECQL4 & c. $1878+1 \mathrm{G}>\mathrm{C}$ & - & donor_splice_site & Not present & 32 & Not present & $\begin{array}{c}\text { Pathogenic } \\
\text { (PVS1;PM2;PP3) }\end{array}$ \\
\hline LLS15 & RAD52 & c. $154 \mathrm{~A}>\mathrm{T}$ & I52L (p.Ile52Leu) & missense & Not present & 25.1 & Not present & VUS (PM2) \\
\hline \multicolumn{9}{|c|}{ Patients with biallelic inactivation in MMR core or their associated genes } \\
\hline LLS18 & PALB2 & c. $2606 \mathrm{C}>\mathrm{T}$ & S869F (p.Ser869Phe) & missense & Not present & 29.2 & VUS & VUS (PM1;PM2;PP3) \\
\hline LLS21 & $U V R A G$ & c. $937 \mathrm{C}>\mathrm{G}$ & Q313E (p.Gln313Glu) & missense & Not present & 26.3 & Not present & VUS (PM2) \\
\hline LLS09 & RAD54L & c. $767-2 \mathrm{~A}>\mathrm{G}$ & - & splice_acceptor & $2.38663 \times 10^{-5}$ & 33 & Not present & VUS (PVS1;PP3) \\
\hline LLS10 & RAD54L & c. $17 \mathrm{C}>\mathrm{T}$ & A6V (p.Ala6Val) & missense & $3.98108 \times 10^{-6}$ & 32 & Not present & VUS (PM2;PP3) \\
\hline LLS02 & MCM2 & c. $364 \mathrm{C}>\mathrm{T}$ & R122W (p.Arg122Trp) & missense & $1.66295 \times 10^{-5}$ & 25.2 & Not present & VUS (PM2;PP3;BP1) \\
\hline
\end{tabular}

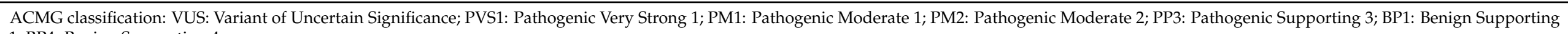
1; BP4: Benign Supporting 4. 
Two different variants (c.767-2A > G and c.17C > T, p.Ala6Val) in RAD54L were identified in two patients (LLS9 and LLS10, respectively); the first variant impacted the helicase ATP-binding domain, and the second one, which was a missense variant, impaired the region of interest related to chromatin remodeling and ATPase activity. Interestingly, both patients had somatic inactivation of $M S H 3$ with associated $\mathrm{LOH}$, and similar TMB and mutational signature profiles.

Lastly, the PALB2 missense variant (c.2606C > T;p.Ser869Phe) was present in LLS18, whose respective tumor showed the largest indel burden. This was the only candidate variant present in Clinvar (as a VUS), and previously reported in a suspected Lynch patient [20].

\section{Discussion}

In this study of a well-annotated cohort of 15 early-onset CRC LLS patients, we performed germline and tumor WES analysis to elucidate the underlying molecular basis of an increasingly common clinical challenge. We identified four cases (27\%) with double somatic alterations in MMR core genes explaining their findings on IHC testing, as well as three additional cases with double $\mathrm{MSH} 3$ somatic alterations in tumors with unexplained MSH2/MSH6 loss of expression, and two cases with potential POLD1 biallelic variants. These results support the idea that MSH3 testing may be considered in CRC samples with unexplained MSH2 loss of IHC expression.

In approximately $30 \%$ of CRC cases [8,21], the presence of MSI or loss of immunochemical expression of MMR genes is found with no corresponding germline pathogenic variants. These LLS patients [9] are clinically represented by at least two different subsets. The first group includes cases in which the clinical characteristics, such as early onset of CRC or a strong family cancer history, suggest a hereditary origin, whereas the second subset includes a significant proportion of LLS families who experience late CRC onset and have no family history of cancer, and therefore, may represent sporadic tumors with no need for preventive measures for themselves or their relatives. LLS appears to be a heterogeneous condition between these two situations.

Testing for somatic variants in MMR genes has been proposed for differential diagnosis between hereditary and sporadic cases in this scenario, and some groups have proposed investigating somatic variants in MMR genes that might explain sporadic CRC cases with LLS [22]. We identified six studies, summarized in Table S1, that have specifically analyzed combinations of biallelic somatic MMR gene variants or single somatic MMR gene variants with associated LOH in patients with CRC and LLS. Sourouille et al. [13], Mensenkamp et al. [14], Geurts-Giele et al. [15], Haraldsdottir et al. [16], and more recently Xicola et al. [23] and Porkka et al. [24], have identified 24\% (4/17), 48\% (11/23), 51\% (17/33), 93\% (14/15), 54\% (6/11), and 79\% (11/14) cases with MMR double somatic alterations, respectively. The $27 \%(4 / 15)$ rate of cases in our study falls within the range of these previous reports and with a similar number of analyzed patients. This very broad range (24\% to $93 \%$ ) of MMR double somatic alterations in CRC LLS patients probably reflects underlying clinical differences between the described cohorts, such as the clear variation in age at CRC diagnosis, ranging from 30 to 65. Of note, Sourouille et al. [13], with a 24\% rate of double somatic alterations, did not analyze $\mathrm{LOH}$ in samples with one MMR somatic variant and is likely missing some cases. Therefore, our cohort could have the lowest percentage of cases with double MMR somatic alterations, coherent with the fact that it presents the youngest age range at CRC diagnosis. Hence, an interesting hypothesis is that the older the age at CRC diagnosis, the higher the probability of double somatic MMR alteration, and the lower the probability of a hereditary origin.

POLE, POLD1, MUTYH, MSH3, MLH3, and PMS1 somaticalterations analysis in our eleven unexplained CRC LLS revealed some interesting findings. We observed double somatic alterations in five of these cases, two presenting biallelic POLD1 variants, and three showing double $\mathrm{MSH} 3$ somatic alterations. 
MSH3 is part of a MutS homolog heterodimer with MSH2, named MutSb, which partially overlaps with MutSa, a heterodimer composed of MSH2 and MSH6 with mispairrecognition specificity [25]. In mouse models, elimination of either MSH6 or MSH3 alone still maintains some functional MMR activity, which is consistent with the persistence of the MutSa or MutSb heterodimer, respectively. In humans, MutSa effectively binds single-base substitution and small (single-base) indel mispairs, while MutSb has a solid affinity for larger indel loops with up to ten unpaired bases [26,27]. Thus, inactivation of MSH3 in human cells not only results in MSI that hold dinucleotide repeats but also results in MSI at certain loci with tetranucleotides repeats, termed EMAST [28]. Furthermore, mouse Msh3 deficiency leads to a partial MMR defect and MSI [29,30].

In our study, we found three patients that showed somatic MSH3 truncating variants associated with LOH, in MSH2-/MSH6- tumors. Although we hypothesize that the MSH3 alterations could be the underlying cause of the MMR inactivation, we cannot fully discard the contrary. Indeed, the mutational signatures associated with these tumors are mostly related to an MMR deficiency. We did not search for EMAST features and, therefore, we cannot differentiate between mono/dinucleotides alterations (associated with MMR deficiency) or polynucleotides alterations (associated with EMAST). However, it is well known that MMR-deficient tumors also shared elevated microsatellite alterations at selected tetranucleotide repeats (EMAST) in previous studies [31]. On the other hand, no somatic mutations or $\mathrm{LOH}$ were found in MSH2 in these patients, and only two MSH6 VUS variants were detected (in LLS09 and LLS10). To our knowledge, a specific mutational signature related to MSH3 deficiency is not yet known, besides the previous evidence that MSH3 deficiency causes EMAST or EMAST with low levels of MSI at loci with dinucleotide repeats in CRC. The COSMIC signatures used in our study do not differentiate between mono or polynucleotide repeats. However, it is likely that the MSH3 mutational signature shares some features with those currently known in the COSMIC database to be associated with MMR defects. LLS09 and LLS10 showed concomitant MSH3/MSH6 and MSH3/POLD1 biallelic alterations, respectively. The $\mathrm{LOH}$ observed in MSH3 argues in favor that they occurred prior to those in MSH6 and POLD1. However, this matter should be taken very cautiously and further investigated in order to draw a more solid conclusion.

Regarding germline CRC predisposition, common MSH3 polymorphisms were significantly associated with CRC and prostate cancer as low-penetrance risk alleles [32-34], and recently high-penetrance pathogenic biallelic germline $\mathrm{MSH} 3$ variants have also been associated with CRC and colorectal polyposis syndromes [35]. Therefore, several lines of evidence support the causal relevance of MSH3 deficiency in the initiation of genetic instability and tumorigenesis.

Finally, CBioPortal findings may represent a means of externally validating our MSH3 observations. We postulate that double $\mathrm{MSH} 3$ somatic alterations may represent a different underlying mechanism for the generation of MSI, with loss of MSH2/MSH6 expression by IHC, at least in CRC. Almost half of the 34 unexplained MMR deficient tumors from the compared cohorts (Table S1) presented MSH2/MSH6 protein loss of expression by IHC; it would be interesting to search for MSH3 somatic alterations in these cohorts to support our findings.

The POLD1 gene has been widely associated with MMR deficient tumors [11] but the evidence is not clear about if there is a direct relation between somatic loss of function POLD1 variants and deficiency of MMR proteins or it is a consequence of a mutator phenotype that impact the MMR genes. In our study, we found that it may be possible that loss of function POLD1 variants associated with $\mathrm{LOH}$ could impair the expression of MMR proteins, which could be the case in LLS9045 and LLS5159 patients. Moreover, these patients also showed mutational signatures associated with POLD1 defects in combination with MMR deficiency signatures but no variants in MMR core genes. Interestingly, LLS5159 had the same variant reported as a germline in a recent study [36] and that patient's tumor also showed hypermutation phenotype but was MSS. The biallelic inactivation of POLD1 in LLS5159 could result in this case in an MSI phenotype. 
Average TMB was significantly higher for LLS cases with double somatic alterations compared to LLS cases without double somatic alterations in the explored genes (MMR, $P O L D 1$, and MSH3) and, surprisingly for tumors left-sided, on the contrary to the reported $[37,38]$. This fact could have potential clinical implications, such as hypermutability and neoantigen-induced immunoreactions, rendering MSI tumors candidates for PD-1 blockade-based immunotherapy [39]. Le, D.T. et al. reported that non-Lynch patients with MMR-deficient tumors responded significantly better than Lynch syndrome patients, but no data are available for LLS tumors specifically. Although the presence of certain particularly immunogenic neoantigens, rather than the TMB, may ultimately determine the response of LLS tumors to PD-1 blockade [40], it would be interesting to explore if differences in tumors with particular somatic alterations reflect different levels of PD-1 blockade responses among patients with CRC LLS.

Almost all patients (14/15), except LLS35604, showed at least one mutational signature related to MMR deficiency. The LLS19 patient had a non-Hodgkin lymphoma four years before her CRC, which showed a major SBS11 contribution. This signature is associated with alkylating agents and therefore suggests that her CRC was a consequence of her previous temozolomide treatment. Our findings are in part in concordance with recent reports of high frequency double somatic alterations in MMR genes among patients who underwent treatment with temozolomide [41]. We believe that in the LLS scenario, the mutational signature approach may be useful in patients with prior malignancies, by revealing if the current CRC is a potential consequence of previous chemotherapy treatments [42].

To continue our analysis we explored the presence of germline pathogenic variants in genes involved in the DNA repair functions. We found 9 candidate genes in 10 patients, of which only POLE has been previously associated with early-onset MSI CRC. ERCC6 and PALB2 have also been related to CRC predisposition but not specifically with LLS $[5,11,43]$. Although the remaining six candidates have not been linked to CRC predisposition, they have been related to cancer development and genome maintenance [44-51]. In any case, further functional studies and replication in additional cohorts will be needed in order to further confirm the identified potential candidates for LLS germline predisposition.

\section{Materials and Methods}

\subsection{Patients}

Fifteen unrelated patients from two institutions (Hospital Udaondo, Buenos Aires, Argentina, and Hospital Clínic, Barcelona, Spain) with CRC diagnosed $\leq 40$ y.o. and LLS were included in this study. Demographic and clinical and pathological characteristics were obtained from patients' medical records, and family history of cancer in first and seconddegree relatives was collected by a personal interview. All patients presented tumors with MSI and/or IHC loss of MSH2, MSH6, PMS2, or loss of MLH1 with somatic wildtype V600EBRAF and no MLH1 methylation, as well as not detected germline pathogenic variants in the MMR genes or EPCAM. Relevant demographic and clinical and pathological features are presented in Table 1. It should be noted that patient LLS06 presented absence of the MSH6 protein expression and MSS in her tumor.

CRC tissue samples and germline DNA were obtained for each patient from the respective institution biobank. Matched tumor and germline DNA samples were used to perform WES when available (15/15 patients) with optimal quantity and quality. A QIAamp Tissue Kit (Qiagen, Redwood City, CA, USA) was used to isolate tumor DNA from formalin-fixed paraffin-embedded (FFPE) tissue following the manufacturer's instructions, achieving $70-80 \%$ tumor cells among all 15 available samples.

This research has been approved by the Institutional Review Board and ethics committee on 22 December 2015. Patients signed a protocol-specific informed consent.

\subsection{Whole Exome Sequencing}

WES was performed in tumor and germline samples of selected patients using the HiSeq2000 platform (Illumina, San Diego, CA, USA) and SureSelectXT Human All Exon v5 
kit (Agilent, Santa Clara, CA, USA) for exon enrichment. Indexed libraries were pooled and massively parallel-sequenced using a paired-end $2 \times 75 \mathrm{bp}$ read length protocol. Quality control of sequencing data was performed in all samples prior to their analysis using the Real-Time Analysis software sequence pipeline (Illumina). Additionally, the proportion of all shared exome regions sequenced with a coverage $\geq 10 \times$ was evaluated for tumor samples.

The Burrows-Wheeler Aligner (BWA-MEM algorithm) was used for read mapping to the human reference genome (build hs37d5, based on NCBI GRCh37). PCR duplicates were discarded using the Mark Duplicates tool (Picard, Broad Institute, Cambridge, MA, USA), and then indel realignment and base quality score recalibration were performed with the Genome Analysis Toolkit (GATK, Broad Institute, Cambridge, MA, USA).

\subsection{Mutational Profiling and Mutational Signature Analysis}

In order to find somatic alterations that could explain the loss of expression pattern, MMR core genes (MLH1, MSH2, MSH6, PMS2), their associated genes MSH3, MLH3, and PMS1, and the additional MUTYH, POLE, and POLD1 genes were considered to search for potentially pathogenic variants and predicting loss of heterozygosis $(\mathrm{LOH})$ using somatic WES. LOH was defined as: (1) variant allele frequency (VAF) for a variant being $>80 \%$ higher than the average VAF of all tumor variants, and (2) corroborated by the analysis of shifts in expected VAF among germline polymorphisms within the same gene region. A putative $\mathrm{LOH}$ was defined as a VAF between $40-80 \%$ higher than the average somatic variant VAF [16]. In Table S3 are shown tumor reads depth and tumor reads from alternative allele and from reference allele.

Additionally, particular tumor features, including tumor mutational burden (TMB) and mutational signatures were analyzed. TMB was described as the total number of single nucleotide variants (SNVs) per megabase $(\mathrm{Mb})$ accumulated in a given sample, assuming that an average WES sample accounts for $30 \mathrm{Mb}$ with acceptable sequencing quality values. De novo mutational signature extraction was performed using SigProfilerExtractor computational framework, based on nonnegative matrix factorization [18]. Activities were calculated after the decomposition of the extracted signatures according to the reference set of mutational signatures described in COSMIC database (Mutational Signatures v3.1-Jun 2020). All data were integrated and visualized with Oviz-Bio platform (https:/ / bio.oviz. org/ access date: 16 January 2021) [52].

\subsection{Variant Calling and Filtering}

The HaplotypeCaller (GATK), MuTect2 (GATK), and Strelka2 (Illumina) were used for SNV and indels calling for germline and tumor samples, respectively. Regarding variant annotation, several databases were considered, including SnpEff and dbNSFP for variant position and pathogenicity annotations. SIFT (prediction of damaging), PolyPhen2 (HumVar prediction of probably damaging or possibly damaging), and CADD (Phred score $\geq 20$ ) were used for pathogenicity prediction of missense variants.

Germline WES data were analyzed using an in-house $\mathrm{R}$ language pipeline described in previous studies [53]. Functions related to CRC or cancer, in general, were prioritized. DNA repair functions were used as the main functional filter.

For tumor SNVs and indels, a similar filtering pipeline was used [54], restraining selected variants to those having a coverage $\geq 10 \times$ both in germline and somatic samples, a tumor VAF $\geq 10 \%$, and also selecting truncating (nonsense, splice site, and frameshift variants) or missense variants fulfilling at least two of the three missense pathogenicity tools criteria. (CADD, Polyphen2, and SIFT).

\subsection{Germline Variant Prioritization and Validation}

From the automatic filtering process performed for all variant types considered, a large number of potentially pathogenic alterations were identified for every sample. Thus, an additional prioritization process was implemented to select truly relevant alterations 
for the phenotype of interest. Using the access to both germline and somatic WES data, an integrated strategy based on Knudson's two-hit hypothesis was developed to search for potential tumor suppressor genes (TSGs) associated with CRC germline predisposition. Genes with a potentially deleterious germline variant (first hit, SNV/indel) and a predicted second mutational event in the tumor (second hit, $\mathrm{SNV}$ /indel or $\mathrm{LOH}$ ) were thus prioritized.

The prioritization process was completed with an additional stringent functional selection of the candidate genes compatible with the TSG model expected. Regarding function of candidate genes, DNA repair was the most stringent filter applied. Candidate genes identified in this process were manually curated according to functional evidence. Particular attention was paid to genes known to be involved in predisposition to CRC and other neoplasms by reviewing data present in OMIM (Online Mendelian Inheritance in Man; http:/ / www.omim.org/ access date: 16 January 2021) and ClinVar (https: / /www. ncbi.nlm.nih.gov / clinvar/ access date: 16 January 2021). Final prioritized variants were validated by manual inspection of the WES data with Integrative Genomics Viewer [55].

\section{Conclusions}

In conclusion, we contribute new insights into the somatic characterization of LLS CRC, postulating MSH3 and POLD1 double somatic alterations as an underlying cause of MSI phenotype in CRCs with unexplained loss of IHC MSH2/MSH6 expression. Although the limited sample size of our study hinders the generalization of our observations and functional validation of our work is critical to provide translational conclusions, we propose intrinsic biological differences between LLS with and without somatic alterations that could facilitate targeted approaches for treatment, and we suggest new germline candidate genes in this scenario that need to be further investigated. We need to join efforts to understand this rare but increasingly common clinical scenario and develop a consensus on the terminology. For instance, should the term LLS include cases with double MMR gene somatic alterations, or not? Are these cases, especially early-onset or with a strong family history of cancer, truly "explained"? As Ladabaum pointed out [56], until we have a detailed molecular understanding of all LLS phenotypes, we need to guide intelligently our patients and their families in order to manage their future cancer risk, by making use of the clinical phenotype, our medical intuition, and common sense.

Supplementary Materials: The following are available online at https:/ / www.mdpi.com/2072-669 4/13/6/1259/s1, Figure S1: Additional most common somatic mutated genes in colorectal cancer, Figure S2: TMB comparison between biallelic somatic inactivations patients and with those who are not biallelic inactivated, Figure S3: Single Base Substitution and InDels De novo mutational signatures, Table S1: CRC Patients from CBioPortal with MSH3 truncating variants and no other MMR alterations showing microsatellite instability, Table S2: Comparative studies showing clinical and molecular differences in published cohorts, Table S3: Additional quality sequencing data from Table 2 variants, Table S4: InSight database classification from somatic MMR variants in Table 2, Table S5: ACMG classification criteria from Table 2 somatic variants.

Author Contributions: Conceptualization, M.G., M.D.-G., S.C.-B. and M.A. investigation, M.G., M.D.-G., L.B., S.F.-E., J.M., M.C. (Miriam Cuatrecasas), T.O., S.I., G.M., M.C. (Marcela Carballido), J.R., D.C., E.R., A.C., F.B., S.C.-B., M.A.; writing-original draft preparation, M.G., M.D.-G., S.C.-B. and M.A.; writing-review and editing, all authors; visualization, M.G. and M.D.-G.; supervision, S.C.-B. and M.A. funding acquisition, F.B., A.C., S.C.-B. and M.A. All authors have read and agreed to the published version of the manuscript.

Funding: M.G. and M.A. were supported by Foundation Nelia et Amadeo Barletta contract. M.D.-G. was supported by a contract from Agència de Gestiód'AjutsUniversitarisi de Recerca -AGAUR(Generalitat de Catalunya, 2019FI_B2_00203). L.B. was supported by a Juan de la Cierva postdoctoral contract (FJCI-2017-32593). J.M. was supported by a contract from CIBEREHD. CIBEREHD is funded by the Instituto de Salud Carlos III. This research was supported by grants from Foundation Nelia et Amadeo Barletta FNAB, Fondo de Investigación Sanitaria/FEDER (17/00878,19/01867, 20/00113), CERCA Program (Generalitat de Catalunya) and Agència de Gestiód'AjutsUniversitarisi de Recerca 
(Generalitat de Catalunya, GRPRE 2017SGR21, GRC 2017SGR653). This article is based upon work from COST Action CA17118, supported by COST (European Cooperation in Science and Technology). www.cost.eu. Access date: 22 February 2021

Institutional Review Board Statement: The study was conducted according to the guidelines of the Declaration of Helsinki, and approved by the Institutional Review Board (or Ethics Committee) of Hospital de Gastroenterología “Dr. Carlos Bonorino Udaondo" (ethic code: HBU-ONCO-LYNCH II, approval date: 22 December 2015).

Informed Consent Statement: Informed consent was obtained from all subjects involved in the study.

Data Availability Statement: Raw data are available upon request.

Acknowledgments: We are sincerely grateful to the patients, CNAG and Biobank of Hospital Clínic-IDIBAPS. The work was carried out (in part) at the Esther Koplowitz Centre and at the Gastroenterology Hospital “Carlos B. Udaondo”.

Conflicts of Interest: The authors declare no conflict of interest.

\section{References}

1. Bray, F.; Ferlay, J.; Soerjomataram, I.; Siegel, R.L.; Torre, L.A.; Jemal, A. Global cancer statistics 2018: GLOBOCAN estimates of incidence and mortality worldwide for 36 cancers in 185 countries. CA Cancer J. Clin. 2018, 68, 394-424. [CrossRef] [PubMed]

2. Siegel, R.L.; Jakubowski, C.D.; Fedewa, S.A.; Davis, A.; Azad, N.S. Colorectal Cancer in the Young: Epidemiology, Prevention, Management. Am. Soc. Clin. Oncol. Educ. Book 2020, 40, 1-14. [CrossRef] [PubMed]

3. Young, J.P.; Win, A.K.; Rosty, C.; Flight, I.; Roder, D.; Young, G.P.; Frank, O.; Suthers, G.K.; Hewett, P.J.; Ruszkiewicz, A.; et al. Rising incidence of early-onset colorectal cancer in Australia over two decades: Report and review. J. Gastroenterol. Hepatol. 2015, 30, 6-13. [CrossRef] [PubMed]

4. Vuik, F.E.; Nieuwenburg, S.A.; Bardou, M.; Lansdorp-Vogelaar, I.; Dinis-Ribeiro, M.; Bento, M.J.; Zadnik, V.; Pellisé, M.; Esteban, L.; Kaminski, M.F.; et al. Increasing incidence of colorectal cancer in young adults in Europe over the last 25 years. Gut 2019, 68, 1820-1826. [CrossRef] [PubMed]

5. Yurgelun, M.B.; Kulke, M.H.; Fuchs, C.S.; Allen, B.A.; Uno, H.; Hornick, J.L.; Ukaegbu, C.I.; Brais, L.K.; McNamara, P.G.; Mayer, R.J.; et al. Cancer Susceptibility Gene Mutations in Individuals With Colorectal Cancer. J. Clin. Oncol. 2017, 35, 1086-1095. [CrossRef]

6. Schmeler, K.M.; Lynch, H.T.; Chen, L.-M.; Munsell, M.F.; Soliman, P.T.; Clark, M.B.; Daniels, M.S.; White, K.G.; Boyd-Rogers, S.G.; Conrad, P.G.; et al. Prophylactic surgery to reduce the risk of gynecologic cancers in the Lynch syndrome. N. Engl. J. Med. 2006, 354, 261-269. [CrossRef]

7. Biller, L.H.; Syngal, S.; Yurgelun, M.B. Recent advances in Lynch syndrome. Fam. Cancer 2019, 18, 211-219. [CrossRef]

8. Antelo, M.; Golubicki, M.; Roca, E.; Mendez, G.; Carballido, M.; Iseas, S.; Cuatrecasas, M.; Moreira, L.; Sanchez, A.; Carballal, S.; et al. Lynch-like syndrome is as frequent as Lynch syndrome in early-onset nonfamilial nonpolyposis colorectal cancer. Int. J. Cancer 2019, 145, 705-713. [CrossRef]

9. Rodríguez-Soler, M.; Pérez-Carbonell, L.; Guarinos, C.; Zapater, P.; Castillejo, A.; Barberá, V.M.; Juárez, M.; Bessa, X.; Xicola, R.M.; Clofent, J.; et al. Risk of cancer in cases of suspected lynch syndrome without germline mutation. Gastroenterology 2013, 144, 926-932.e1, quiz e13-e14. [CrossRef]

10. Morak, M.; Heidenreich, B.; Keller, G.; Hampel, H.; Laner, A.; de la Chapelle, A.; Holinski-Feder, E. Biallelic MUTYH mutations can mimic Lynch syndrome. Eur. J. Hum. Genet. 2014, 22, 1334-1337. [CrossRef] [PubMed]

11. Elsayed, F.A.; Kets, C.M.; Ruano, D.; van den Akker, B.; Mensenkamp, A.R.; Schrumpf, M.; Nielsen, M.; Wijnen, J.T.; Tops, C.M.; Ligtenberg, M.J.; et al. Germline variants in POLE are associated with early onset mismatch repair deficient colorectal cancer. Eur. J. Hum. Genet. 2015, 23, 1080-1084. [CrossRef] [PubMed]

12. Jansen, A.M.; van Wezel, T.; van den Akker, B.E.; Ventayol Garcia, M.; Ruano, D.; Tops, C.M.; Wagner, A.; Letteboer, T.G.; Gómez-García, E.B.; Devilee, P.; et al. Combined mismatch repair and POLE/POLD1 defects explain unresolved suspected Lynch syndrome cancers. Eur. J. Hum. Genet. 2016, 24, 1089-1092. [CrossRef] [PubMed]

13. Sourrouille, I.; Coulet, F.; Lefevre, J.H.; Colas, C.; Eyries, M.; Svrcek, M.; Bardier-Dupas, A.; Parc, Y.; Soubrier, F. Somatic mosaicism and double somatic hits can lead to MSI colorectal tumors. Fam. Cancer 2013, 12, 27-33. [CrossRef]

14. Mensenkamp, A.R.; Vogelaar, I.P.; van Zelst-Stams, W.A.G.; Goossens, M.; Ouchene, H.; Hendriks-Cornelissen, S.J.B.; Kwint, M.P.; Hoogerbrugge, N.; Nagtegaal, I.D.; Ligtenberg, M.J.L. Somatic mutations in MLH1 and MSH2 are a frequent cause of mismatch-repair deficiency in Lynch syndrome-like tumors. Gastroenterology 2014, 146, 643-646.e8. [CrossRef]

15. Geurts-Giele, W.R.R.; Leenen, C.H.M.; Dubbink, H.J.; Meijssen, I.C.; Post, E.; Sleddens, H.F.B.M.; Kuipers, E.J.; Goverde, A.; van den Ouweland, A.M.W.; van Lier, M.G.F.; et al. Somatic aberrations of mismatch repair genes as a cause of microsatellite-unstable cancers. J. Pathol. 2014, 234, 548-559. [CrossRef] 
16. Haraldsdottir, S.; Hampel, H.; Tomsic, J.; Frankel, W.L.; Pearlman, R.; de la Chapelle, A.; Pritchard, C.C. Colon and endometrial cancers with mismatch repair deficiency can arise from somatic, rather than germline, mutations. Gastroenterology 2014, 147, 1308-1316.e1. [CrossRef] [PubMed]

17. Tate, J.G.; Bamford, S.; Jubb, H.C.; Sondka, Z.; Beare, D.M.; Bindal, N.; Boutselakis, H.; Cole, C.G.; Creatore, C.; Dawson, E.; et al. COSMIC: The Catalogue Of Somatic Mutations In Cancer. Nucleic Acids Res. 2019, 47, D941-D947. [CrossRef]

18. Alexandrov, L.B.; Kim, J.; Haradhvala, N.J.; Huang, M.N.; Tian Ng, A.W.; Wu, Y.; Boot, A.; Covington, K.R.; Gordenin, D.A.; Bergstrom, E.N.; et al. The repertoire of mutational signatures in human cancer. Nature 2020, 578, 94-101. [CrossRef]

19. Golubicki, M.; Bonjoch, L.; Acuña-Ochoa, J.G.; Díaz-Gay, M.; Muñoz, J.; Cuatrecasas, M.; Ocaña, T.; Iseas, S.; Mendez, G.; Cisterna, D.; et al. Germline biallelic Mcm8 variants are associated with early-onset Lynch-like syndrome. JCI Insight 2020, 5. [CrossRef]

20. Yurgelun, M.B.; Allen, B.; Kaldate, R.R.; Bowles, K.R.; Judkins, T.; Kaushik, P.; Roa, B.B.; Wenstrup, R.J.; Hartman, A.-R.; Syngal, S. Identification of a Variety of Mutations in Cancer Predisposition Genes in Patients With Suspected Lynch Syndrome. Gastroenterology 2015, 149, 604-613.e20. [CrossRef]

21. Pérez-Carbonell, L.; Ruiz-Ponte, C.; Guarinos, C.; Alenda, C.; Payá, A.; Brea, A.; Egoavil, C.M.; Castillejo, A.; Barberá, V.M.; Bessa, $X$.; et al. Comparison between universal molecular screening for Lynch syndrome and revised Bethesda guidelines in a large population-based cohort of patients with colorectal cancer. Gut 2012, 61, 865-872. [CrossRef] [PubMed]

22. Hampel, H.; Pearlman, R.; Beightol, M.; Zhao, W.; Jones, D.; Frankel, W.L.; Goodfellow, P.J.; Yilmaz, A.; Miller, K.; Bacher, J.; et al. Assessment of Tumor Sequencing as a Replacement for Lynch Syndrome Screening and Current Molecular Tests for Patients With Colorectal Cancer. JAMA Oncol. 2018, 4, 806-813. [CrossRef]

23. Xicola, R.M.; Clark, J.R.; Carroll, T.; Alvikas, J.; Marwaha, P.; Regan, M.R.; Lopez-Giraldez, F.; Choi, J.; Emmadi, R.; AlagiozianAngelova, V.; et al. Implication of DNA repair genes in Lynch-like syndrome. Fam. Cancer 2019, 18, 331-342. [CrossRef] [PubMed]

24. Porkka, N.; Lahtinen, L.; Ahtiainen, M.; Böhm, J.P.; Kuopio, T.; Eldfors, S.; Mecklin, J.-P.; Seppälä, T.T.; Peltomäki, P. Epidemiological, clinical and molecular characterization of Lynch-like syndrome: A population-based study. Int. J. Cancer 2019, 145, 87-98. [CrossRef] [PubMed]

25. Srivatsan, A.; Bowen, N.; Kolodner, R.D. Mispair-specific recruitment of the Mlh1-Pms1 complex identifies repair substrates of the Saccharomyces cerevisiae Msh2-Msh3 complex. J. Biol. Chem. 2014, 289, 9352-9364. [CrossRef]

26. Haugen, A.C.; Goel, A.; Yamada, K.; Marra, G.; Nguyen, T.-P.; Nagasaka, T.; Kanazawa, S.; Koike, J.; Kikuchi, Y.; Zhong, X.; et al. Genetic instability caused by loss of MutS homologue 3 in human colorectal cancer. Cancer Res. 2008, 68, 8465-8472. [CrossRef]

27. Plaschke, J.; Preußler, M.; Ziegler, A.; Schackert, H.K. Aberrant protein expression and frequent allelic loss of MSH3 in colorectal cancer with low-level microsatellite instability. Int. J. Colorectal Dis. 2012, 27, 911-919. [CrossRef]

28. Hile, S.E.; Shabashev, S.; Eckert, K.A. Tumor-specific microsatellite instability: Do distinct mechanisms underlie the MSI-L and EMAST phenotypes? Mutat. Res. 2013, 743-744, 67-77. Mutat. Res. 2013, 743-744, 67-77. [CrossRef]

29. Edelmann, W.; Umar, A.; Yang, K.; Heyer, J.; Kucherlapati, M.; Lia, M.; Kneitz, B.; Avdievich, E.; Fan, K.; Wong, E.; et al. The DNA mismatch repair genes Msh3 and Msh6 cooperate in intestinal tumor suppression. Cancer Res. 2000, 60, 803-807.

30. Hegan, D.C.; Narayanan, L.; Jirik, F.R.; Edelmann, W.; Liskay, R.M.; Glazer, P.M. Differing patterns of genetic instability in mice deficient in the mismatch repair genes Pms2, Mlh1, Msh2, Msh3 and Msh6. Carcinogenesis 2006, 27, 2402-2408. [CrossRef] [PubMed]

31. Watson, M.M.; Lea, D.; Rewcastle, E.; Hagland, H.R.; Søreide, K. Elevated microsatellite alterations at selected tetranucleotides in early-stage colorectal cancers with and without high-frequency microsatellite instability: Same, same but different? Cancer Med. 2016, 5, 1580-1587. [CrossRef]

32. Orimo, H.; Nakajima, E.; Yamamoto, M.; Ikejima, M.; Emi, M.; Shimada, T. Association between single nucleotide polymorphisms in the hMSH3 gene and sporadic colon cancer with microsatellite instability. J. Hum. Genet. 2000, 45, 228-230. [CrossRef]

33. Berndt, S.I.; Platz, E.A.; Fallin, M.D.; Thuita, L.W.; Hoffman, S.C.; Helzlsouer, K.J. Mismatch repair polymorphisms and the risk of colorectal cancer. Int. J. Cancer 2007, 120, 1548-1554. [CrossRef]

34. Hirata, H.; Hinoda, Y.; Kawamoto, K.; Kikuno, N.; Suehiro, Y.; Okayama, N.; Tanaka, Y.; Dahiya, R. Mismatch repair gene MSH3 polymorphism is associated with the risk of sporadic prostate cancer. J. Urol. 2008, 179, 2020-2024. [CrossRef]

35. Adam, R.; Spier, I.; Zhao, B.; Kloth, M.; Marquez, J.; Hinrichsen, I.; Kirfel, J.; Tafazzoli, A.; Horpaopan, S.; Uhlhaas, S.; et al. Exome Sequencing Identifies Biallelic MSH3 Germline Mutations as a Recessive Subtype of Colorectal Adenomatous Polyposis. Am. J. Hum. Genet. 2016, 99, 337-351. [CrossRef]

36. Mur, P.; García-Mulero, S.; Del Valle, J.; Magraner-Pardo, L.; Vidal, A.; Pineda, M.; Cinnirella, G.; Martín-Ramos, E.; Pons, T.; López-Doriga, A.; et al. Role of POLE and POLD1 in familial cancer. Genet. Med. 2020. [CrossRef]

37. Salem, M.E.; Weinberg, B.A.; Xiu, J.; El-Deiry, W.S.; Hwang, J.J.; Gatalica, Z.; Philip, P.A.; Shields, A.F.; Lenz, H.-J.; Marshall, J.L. Comparative molecular analyses of left-sided colon, right-sided colon, and rectal cancers. Oncotarget 2017, 8, 86356-86368. [CrossRef]

38. Salem, M.E.; Puccini, A.; Grothey, A.; Raghavan, D.; Goldberg, R.M.; Xiu, J.; Korn, W.M.; Weinberg, B.A.; Hwang, J.J.; Shields, A.F.; et al. Landscape of Tumor Mutation Load, Mismatch Repair Deficiency, and PD-L1 Expression in a Large Patient Cohort of Gastrointestinal Cancers. Mol. Cancer Res. 2018, 16, 805-812. [CrossRef] [PubMed]

39. Le, D.T.; Uram, J.N.; Wang, H.; Bartlett, B.R.; Kemberling, H.; Eyring, A.D.; Skora, A.D.; Luber, B.S.; Azad, N.S.; Laheru, D.; et al. PD-1 Blockade in Tumors with Mismatch-Repair Deficiency. N. Engl. J. Med. 2015, 372, 2509-2520. [CrossRef] [PubMed] 
40. Dudley, J.C.; Lin, M.-T.; Le, D.T.; Eshleman, J.R. Microsatellite Instability as a Biomarker for PD-1 Blockade. Clin. Cancer Res. 2016, 22, 813-820. [CrossRef] [PubMed]

41. Rigter, L.S.; Snaebjornsson, P.; Rosenberg, E.H.; Atmodimedjo, P.N.; Aleman, B.M.; Ten Hoeve, J.; Geurts-Giele, W.R.; van Ravesteyn, T.W.; Hoeksel, J.; PALGA Group; et al. Double somatic mutations in mismatch repair genes are frequent in colorectal cancer after Hodgkin's lymphoma treatment. Gut 2018, 67, 447-455. [CrossRef] [PubMed]

42. Pich, O.; Muiños, F.; Lolkema, M.P.; Steeghs, N.; Gonzalez-Perez, A.; Lopez-Bigas, N. The mutational footprints of cancer therapies. Nat. Genet. 2019, 51, 1732-1740. [CrossRef]

43. Arora, S.; Yan, H.; Cho, I.; Fan, H.-Y.; Luo, B.; Gai, X.; Bodian, D.L.; Vockley, J.G.; Zhou, Y.; Handorf, E.A.; et al. Genetic Variants That Predispose to DNA Double-Strand Breaks in Lymphocytes From a Subset of Patients With Familial Colorectal Carcinomas. Gastroenterology 2015, 149, 1872-1883.e9. [CrossRef]

44. Kohzaki, M.; Ootsuyama, A.; Sun, L.; Moritake, T.; Okazaki, R. Human RECQL4 represses the RAD52-mediated single-strand annealing pathway after ionizing radiation or cisplatin treatment. Int. J. Cancer 2020, 146, 3098-3113. [CrossRef] [PubMed]

45. Das, R.; Kundu, S.; Laskar, S.; Choudhury, Y.; Ghosh, S.K. Assessment of DNA repair susceptibility genes identified by whole exome sequencing in head and neck cancer. DNA Repair 2018, 66-67, 50-63. DNA Repair 2018, 66-67, 50-63. [CrossRef]

46. Ui, A.; Chiba, N.; Yasui, A. Relationship among DNA double-strand break (DSB), DSB repair, and transcription prevents genome instability and cancer. Cancer Sci. 2020, 111, 1443-1451. [CrossRef] [PubMed]

47. Knijnenburg, T.A.; Wang, L.; Zimmermann, M.T.; Chambwe, N.; Gao, G.F.; Cherniack, A.D.; Fan, H.; Shen, H.; Way, G.P.; Greene, C.S.; et al. Genomic and Molecular Landscape of DNA Damage Repair Deficiency across The Cancer Genome Atlas. Cell Rep. 2018, 23, 239-254.e6. [CrossRef]

48. Fei, L.; Xu, H. Role of MCM2-7 protein phosphorylation in human cancer cells. Cell Biosci. 2018, 8, 43. [CrossRef]

49. Mazin, A.V.; Mazina, O.M.; Bugreev, D.V.; Rossi, M.J. Rad54, the motor of homologous recombination. DNA Repair 2010, 9 , 286-302. [CrossRef]

50. Quach, C.; Song, Y.; Guo, H.; Li, S.; Maazi, H.; Fung, M.; Sands, N.; O'Connell, D.; Restrepo-Vassalli, S.; Chai, B.; et al. A truncating mutation in the autophagy gene UVRAG drives inflammation and tumorigenesis in mice. Nat. Commun. 2019, 10, 5681. [CrossRef]

51. Ali, S.; Zhang, Y.; Zhou, M.; Li, H.; Jin, W.; Zheng, L.; Yu, X.; Stark, J.M.; Weitzel, J.N.; Shen, B. Functional deficiency of DNA repair gene EXO5 results in androgen-induced genomic instability and prostate tumorigenesis. Oncogene 2020, 39, 1246-1259. [CrossRef] [PubMed]

52. Jia, W.; Li, H.; Li, S.; Chen, L.; Li, S.C. Oviz-Bio: A web-based platform for interactive cancer genomics data visualization. Nucleic Acids Res. 2020, 48, W415-W426. [CrossRef] [PubMed]

53. Esteban-Jurado, C.; Vila-Casadesús, M.; Garre, P.; Lozano, J.J.; Pristoupilova, A.; Beltran, S.; Muñoz, J.; Ocaña, T.; Balaguer, F.; López-Cerón, M.; et al. Whole-exome sequencing identifies rare pathogenic variants in new predisposition genes for familial colorectal cancer. Genet. Med. 2015, 17, 131-142. [CrossRef]

54. Díaz-Gay, M.; Franch-Expósito, S.; Arnau-Collell, C.; Park, S.; Supek, F.; Muñoz, J.; Bonjoch, L.; Gratacós-Mulleras, A.; SánchezRojas, P.A.; Esteban-Jurado, C.; et al. Integrated Analysis of Germline and Tumor DNA Identifies New Candidate Genes Involved in Familial Colorectal Cancer. Cancers 2019, 11, 362. [CrossRef]

55. Thorvaldsdóttir, H.; Robinson, J.T.; Mesirov, J.P. Integrative Genomics Viewer (IGV): High-performance genomics data visualization and exploration. Brief. Bioinform. 2013, 14, 178-192. [CrossRef]

56. Ladabaum, U. What Is Lynch-like Syndrome and How Should We Manage It? Clin. Gastroenterol. Hepatol. 2020, 18, 294-296. [CrossRef] [PubMed] 